\title{
Chaine d'instrumentation météorologique : du composant au logiciel
}

\author{
Emilie Péry ${ }^{1}$, Laurent Malaterre ${ }^{2}$ \\ emilie.pery@udamail.fr, laurent.malaterre@univ-bpclermont.fr
}

Adresses :

(1) IUT Département Mesures Physiques - 5 av. Blaise Pascal - BP86 - 63172 Aubière CEDEX

(2) Institut Pascal - 24 av. des Landais - 63171 Aubière CEDEX

\begin{abstract}
RESUME : Cet article décrit une chaine d'instrumentation météorologique complète allant de l'analyse des composants au prototypage sous LabVIEW. Pendant trois séances de T.P. de quatre heures, les étudiants de la licence professionnelle Sciences de la Mesure et du Contrôle réalisent un enregistrement automatique des données météos à l'aide d'une platine intégrant quatre capteurs et de l'ensemble instrumenté par une interface DAQ USB-6008 et LabVIEW. Les objectifs d'apprentissage de ce T.P. s'appuient sur la taxonomie de Bloom, modèle pédagogique qui peut être utilisé dans d'autres enseignements. Ce T.P. est proche des conditions que les étudiants rencontreront dans leur vie professionnelle. Le retour d'expérience mené auprès de ces étudiants a souligné l'attrait de l'autonomie relative, de l'échange et de la variété technique dans un T.P.
\end{abstract}

Mots clés : instrumentation météo, électronique, chaine de mesures, acquisition de données, LabVIEW, taxonomie de Bloom.

\section{INTRODUCTION}

Le besoin de techniciens qualifiés aux problématiques de l'instrumentation au niveau licence est important : c'est dans ce cadre que s'inscrit la licence professionnelle Science de la Mesure et du Contrôle (SiMCo), licence réalisée au département Mesures Physiques de l'IUT de Clermont-Ferrand. Cette licence accueille des étudiants ayant des formations antérieures diversifiées (DUT, BTS, L2 Sciences et Technologie...).

Les objectifs de cette licence sont de former des techniciens supérieurs en science de la mesure et du contrôle capables de :

- gérer des processus industriels,

- mettre en place une chaine de mesure (choix des capteurs...),

- assurer la gestion et l'étalonnage du parc des instruments (vérification de la capabilité des appareils, identification et calcul des incertitudes de mesure...),

- déterminer les paramètres à mesurer, créer les procédures de contrôle,

- réaliser des contrôles non destructifs.

Les compétences acquises lors de cette formation permettent aux futurs diplômés d'avoir un emploi dans les services $\mathrm{R} \& \mathrm{D}$, production, contrôle ou d'être les animateurs, les responsables de la fonction qualité métrologie dans les PME/PMI ou des collaborateurs du service métrologie des grandes entreprises.
Les disciplines de l'EEA ne constituent pas le cœur de métier des étudiants de la licence professionnelle SiMCo mais elles sont utilisées comme outils pour l'instrumentation, instrumentation couvrant l'ensemble des domaines techniques allant de la physique à l'informatique en passant par l'optique. Il faut donc suivre les évolutions des techniques de la mesure et de l'instrumentation pour accompagner des nonspécialistes de l'informatique et de l'électronique. C'est dans ce cadre que nous avons développé un TP balayant l'ensemble de la chaine d'acquisition.

Le but de ce T.P., réparti sur trois séances de quatre heures, est de travailler sur une chaine d'instrumentation complète, ouverte et modulable allant du capteur jusqu'à l'exploitation des mesures. Les étudiants sont amenés à appréhender les pièges de la mise en œuvre d'une chaine électronique d'instrumentation (acquisition et restitution) en vue du traitement et de l'utilisation des mesures par un système informatique.

La maquette, qui a été entièrement conçue et réalisée par nos soins, est présentée dans la première partie de cet article. La deuxième partie montre les capteurs mis à disposition des étudiants et comment ils sont exploités lors du T.P. Enfin, les objectifs pédagogiques, l'évaluation et le retour d'expérience sont discutés dans la troisième et dernière partie. 


\section{MAQUETTE METEOROLOGIQUE}

\subsection{Présentation générale}

Durant les trois séances, les étudiants œuvrent successivement sur les quatre capteurs qu'il est proposé d'étudier :

- Capteur 1 : sonde de température,

- Capteur 2 : composant luxmètre,

- Capteur 3 : baromètre,

- Capteur 4 : centrale anémomètre et girouette.

Ils disposent d'une carte électronique qui héberge ces capteurs avec l'électronique de conditionnement, d'un module d'acquisition prêt à l'emploi et d'un logiciel d'interfaçage sur un ordinateur distant (Figure 1).

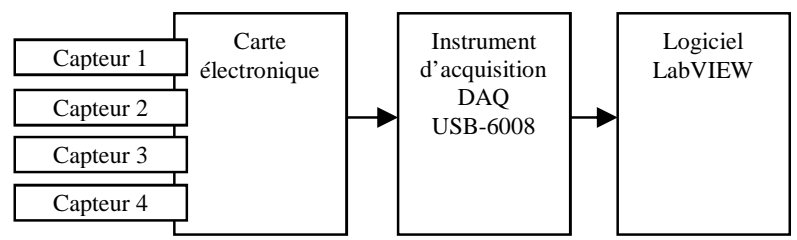

Fig.1 : Diagramme général de la maquette météorologique.

\subsection{Carte électronique}

Dans le respect de la ligne directrice pédagogique, l'ensemble matériel a suivi un schéma de conception adapté. Les contraintes industrielles intervenant habituellement dans l'élaboration de la structure fonctionnelle, la schématique et le routage sont exemptes: d'une part avec un dimensionnement matériel accessible et d'autre part avec l'utilisation de composants électroniques communs (largement distribués et peu chers) à la place de composants spécifiques (aux caractéristiques plus avancés et donc plus onéreux).

\subsubsection{Contraintes}

La réalisation matérielle de la carte météo, orientée pédagogie, respecte les exigences suivantes :

- Aération de l'implantation des composants, présence de picots de tests accessibles et sérigraphie de repérage coordonnée avec la documentation. Cette dernière caractéristique permettant à l'étudiant de facilement localiser les zones d'intervention.

- Doublement de la disponibilité des capteurs par leur implantation locale sur la carte mais aussi par leur déport via une connectique externe.

- Modularité du plan en cours de T.P. par l'implantation de connectique mezzanine et commutateurs switch dual in line (DIL). Ces derniers apportent une alternative de câblage et permet d'interchanger une fonction électronique dans la chaine de mesure.

- Lecture claire du plan technique par rapport au plan fonctionnel.

\subsubsection{Vue fonctionnelle}

La vue fonctionnelle de la carte météo est présentée Figure 2.

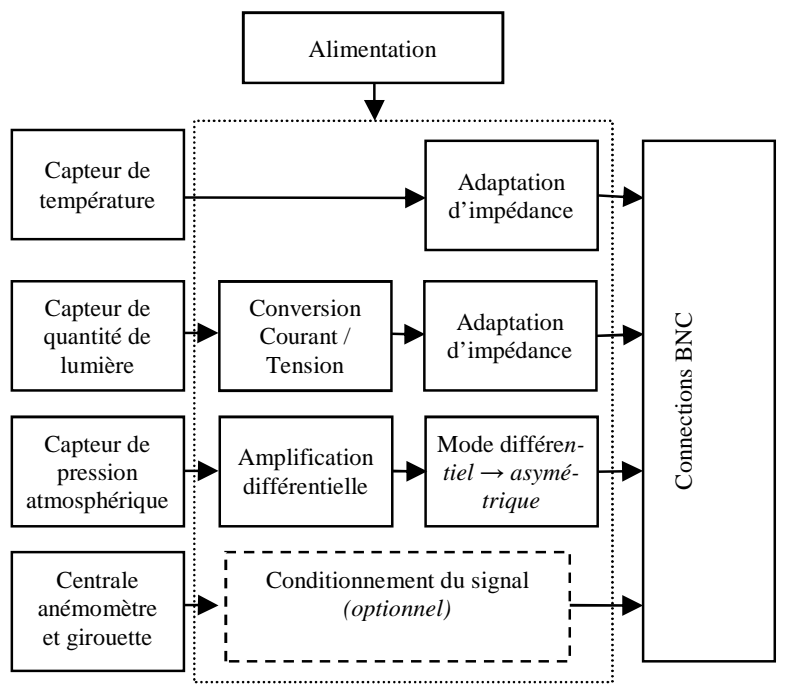

Fig. 2 : Vue fonctionnelle de la carte électronique.

\subsubsection{Réalisation}

La carte météo est câblée sur un support double face avec des composants montés en surface. Les picots de test sont facilement accessibles aux sondes et la connectique externe est adaptée au matériel de T.P. (prises $\mathrm{BNC}$ ). La présence d'une sérigraphie facilite l'identification des éléments physiques sur le schéma et vice-versa. Le changement de plan s'opère par la combinaison de positions d'interrupteurs DIL quatre positions câblées sur la carte : l'étudiant peut ainsi facilement tester des configurations électroniques différentes.

\subsection{Module d'acquisition des données et logiciel}

L'ensemble matériel est interfacé par un module d'acquisition de données multifonction DAQ USB6008 de National Instruments ${ }^{\circledR}$ [1]. Ce module propose plusieurs types d'entrées/sorties d'instrumentation dont 8 entrées vers des convertisseurs analogique/numérique à 10 kéch./s et 12 bits de résolution. La plage de tension mesurée sur une entrée analogique du DAQ USB6008 étant de $\pm 10 \mathrm{~V}$, la résolution en tension du système convertisseur est donc de $4,88 \mathrm{mV}$ et la résolution temporelle des échantillons et de $0,1 \mathrm{~ms}$. Les données acquises sont acheminées vers le logiciel de traitement et de présentation sur ordinateur (LabVIEW@) via une connectique USB.

LabVIEW, commercialisé par National Instruments, est un ensemble de développement intégré logiciel et permet un interfaçage quasi automatique entre le matériel et le traitement de l'information. Le paramétrage logiciel de l'instrument d'acquisition permet d'agir sur la fréquence d'acquisition et le nombre des échantillons par paquets de transfert. La conception s'articule dès 
lors entre une interface graphique ou face avant (fenêtre interactive avec l'utilisateur) et un diagramme (feuille de conception logicielle graphique). La face avant et le diagramme composent un Instrument Virtuel ou VI. Dans les deux cas, les composants virtuels ou logiciels sont soit pris dans une des bibliothèques, soit programmés.

\section{MISE EN OEUVRE}

\subsection{Contexte}

Le but pédagogique de ce T.P. est de procéder à l'instrumentation complète de la chaine de mesure de plusieurs capteurs. Ce T.P. conduit l'étudiant à opérer au plus près du matériel qui est un circuit électronique matériellement ouvert et documenté et à appréhender :

- La recherche ouverte d'informations techniques (livres [2] [3], sites internet, cours...),

- Le vocabulaire associé à la chaine de mesure,

- L'analyse de composants et schémas techniques électroniques,

- Les pièges électroniques et logiciels de l'instrumentation,

- Le prototypage rapide matériel et logiciel d'une mesure sous LabVIEW,

- Les techniques de traitement du signal comme le filtrage.

La Figure 3 présente une vue d'ensemble de la maquette électronique et du boîtier d'acquisition DAQ USB-6008.

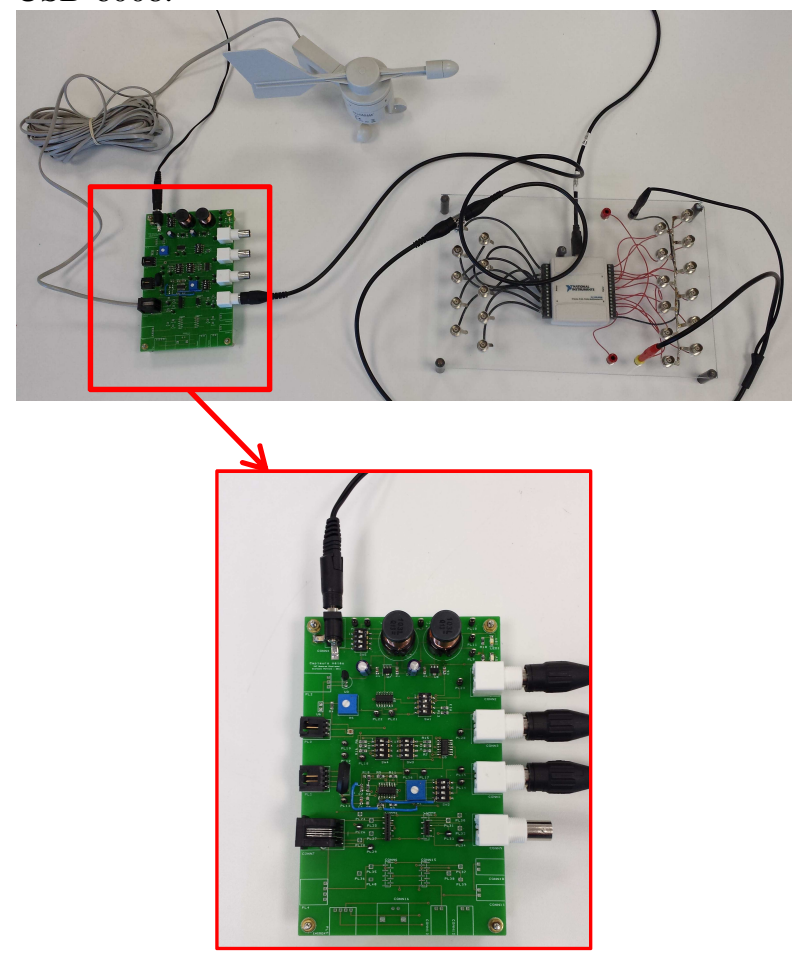

Fig. 3 : Maquette T.P. météo.

\subsection{Mesure de la température}

- Capteur :

La température ambiante est mesurée grâce au capteur LM335 qui délivre une tension évoluant en fonction de la température $\left(10 \mathrm{mV} /{ }^{\circ} \mathrm{K}\right)[4]$.

- Traitement électronique et conditionnement :

Un dispositif de réglage à base de potentiomètre et de résistances, qui sont accessibles sur la platine électronique, permet d'étalonner cette mesure. L'étudiant prend alors connaissance d'un des problèmes électroniques de la mesure à savoir celui dû à la mauvaise adaptation d'impédance entre les sous-ensembles électroniques. En effet, l'étage de sortie du bloc capteur est de l'ordre de $10 \mathrm{k} \Omega$, l'impédance des entrées analogiques étant de $144 \mathrm{k} \Omega$. Dans le cadre des régimes de fonctionnement des dipôles actifs, nous recherchons un maximum de fidélité en tension entre les deux étages. Pour cela, il faut avoir un rapport très faible d'impédance de sortie du dipôle générateur sur celle du dipôle récepteur. Dans notre cas, nous avons un rapport de $1 / 14^{\text {ème }}$. La mesure est alors entachée d'une erreur qu'il faut chercher à corriger. La solution consiste à intercaler un montage à base d'amplificateur opérationnel (AOP) monté en suiveur. Grâce à l'alternative câblée, l'étudiant peut tester les deux configurations et constater la différence entre les valeurs mesurées.

- Traitement logiciel et visualisation des résultats : L'environnement de LabVIEW permet la visualisation directe et en temps réel des mesures. Il est demandé à l'étudiant de procéder logiciellement à la transformation de la valeur en tension de la température (en degrés Kelvin) en une lecture directe en degrés Celsius. Le signal est bruité (environnement électromagnétique perturbé), il faut ajouter un filtrage numérique afin de lisser la mesure.

\subsection{Mesure de la quantité de lumière : le luxmètre}

\section{- Capteur:}

En premier lieu, il convient de faire apprécier par l'étudiant des capacités du capteur. Il s'agit d'un capteur de quantité de lumière (exprimée en lux) SFH 5711 d'OSRAM, qui fournit l'information en courant $i_{\text {out }}$ et dont la réponse (rapide) en fonction de la quantité de lumière est de forme logarithmique (cf. équation 1)[5].

$$
i_{\text {out }}=S \log \frac{E_{v}}{E_{0}}
$$

avec $S$ la sensibilité du capteur $(\mu \mathrm{A}), E_{v}$ le flux de lumière (lux) et $\mathrm{E}_{0}$ le flux de lumière de référence (lux).

L'étudiant doit alors appréhender son utilisation non pas dans le cadre d'une mesure exacte mais plutôt dans le déclenchement à partir d'un seuil (entre 200 et 400 lux) d'évènements rapides. Ce sont des conditions typiques de test de déclenchement de flash photographique, ou de commande automatique de feux automobiles. L'étalonnage du capteur est possible à l'aide d'un luxmètre étalon. 
- Traitement électronique et conditionnement :

Sur le schéma électronique fourni en TP, l'étudiant doit reconnaitre deux étages. Le premier étage dit «Amplification » constitue un convertisseur courant-tension qui établit la relation : $V_{\text {out } / V}=-\left(R_{/ \Omega} I_{/ A}\right)$. Le second étage correspond à un circuit d'adaptation d'impédance à base d'amplificateur opérationnel. A cette fin de déduction, la plupart des sources d'information sont accessibles à l'étudiant (internet, livres techniques, ses cours, ses propres connaissances).

- Traitement logiciel et visualisation des résultats : Les échantillons en tension sont traduits en lux par l'intermédiaire de l'équation fournie par la documentation technique et l'analyse du schéma électronique de conditionnement du signal. A partir de cette information, l'étudiant programme l'affichage d'un avertisseur tout ou rien autour d'un seuil de quantité de lumière librement défini. Par ailleurs, l'analyse en mode oscilloscope de l'affichage des échantillons sur une durée courte de $100 \mathrm{~ms}$ lui permet de déduire l'origine d'une variation de lumière à $100 \mathrm{~Hz}$ : il s'agit de la variation de l'amplitude de lumière causée par la charge/décharge des néons de la salle de T.P., l'exemple contraire lui étant fourni par la lumière provenant de l'extérieur (émission continue).

\subsection{Mesure de la pression atmosphérique : le ba- romètre}

- Capteur:

Le capteur barométrique MPX2200 mesure la pression atmosphérique entre 0 et 2 bars. Il fournit une mesure en tension différentielle entre 0 et $10 \mathrm{~V}$ [6].

- Traitement électronique et conditionnement :

L'analyse du schéma doit orienter l'étudiant vers la reconnaissance de deux blocs distincts. Le premier est dit d'amplificateur de mesure à base d'AOP. L'étudiant doit en calculer le gain théorique $G=1+2 \frac{R_{1}}{R_{2}}$ et procéder au calibrage pour fournir $1 \mathrm{~V}$ pour $1000 \mathrm{hPa}$ ou 1 bar. Le deuxième bloc permet de convertir l'information différentielle en information en mode commun tout en fournissant une adaptation d'impédance par la même occasion. L'étudiant a le choix d'interfacer la mesure selon ces deux modes (commun ou différentiel) et procéder au câblage matériel et logiciel adéquat.

- Traitement logiciel et visualisation des résultats : La réalisation logicielle conduit ici à seulement filtrer et afficher la valeur à l'instar de la mesure de température décrite plus haut.

\subsection{Mesures anémométriques : vitesse et direction du vent}

Cette partie du T.P. s'inspire du travail de décodage du protocole de communication de la centrale anémomètre girouette TX20 de LaCrosse Technology par un inconnu de la toile [7].
- Capteur:

Le TX20 est un ensemble intégré fournissant les deux mesures de vitesse et de direction du vent. C'est un capteur intelligent qui pré-traite l'information et la fournit numériquement sur un bus deux fils. Le détail du protocole est fourni à l'étudiant qui doit en premier lieu l'analyser physiquement pour ensuite en extraire l'information à l'aide d'un VI LabVIEW.

- Traitement et conditionnement :

La lecture du comportement électronique du signal, à l'aide d'un oscilloscope, doit guider la stratégie de capture des données, stratégie que l'étudiant doit déployer. Les trames sont codées sur un fil de communication série.

- Les trames périodiques $(2,1 \mathrm{~s})$ et numériques envoyées par le capteur influent sur le choix précis de la quantité et de la fréquence de l'échantillonnage.

- Le signal est dégradé par une grande longueur de câble. La solution de l'acquisition par une entrée numérique étant une fausse piste en l'état, l'étudiant doit alors envisager une acquisition analogique. Cependant, la solution d'acquisition par une entrée numérique pourrait être mise en place en insérant un étage de mise en forme du signal (prévu mais pas encore implémenté).

- La recherche des échantillons nécessaires pour reconstruire l'information capteur (composée de mots numériques : un mot de 4 bits et un de 11 bits) est guidée par l'étalement temporel des successions de niveaux logiques, lesquels représentant le bit d'information (slot). Elle prend en compte la durée de ces slots et la synchronisation électronique du signal (front montant du premier slot). Le câblage prend alors la forme de la Figure 4.

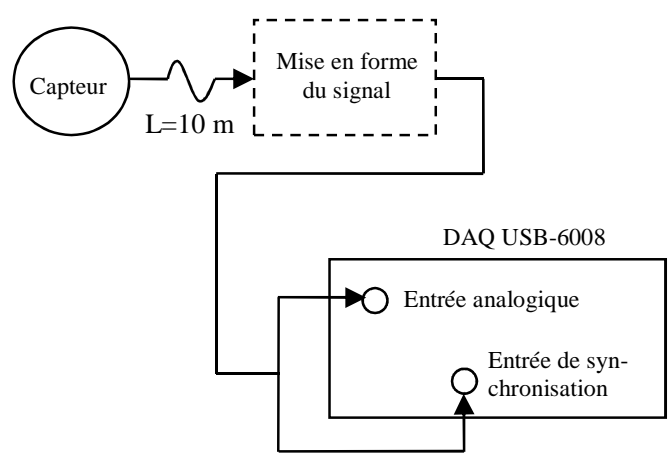

Fig. 4 : Diagramme de câblage.

Cette partie du T.P. mène l'étudiant à adopter une stratégie de mesure à partir d'informations qu'il a luimême déduites grâce à une première approche expérimentale de la maquette et de la documentation.

- Traitement logiciel et visualisation des résultats : Le VI de traitement et d'affichage se résume à reconvertir la séquence de niveaux analogiques en mots 
numériques. Le cheminement séquentiel du traitement de l'information suit alors le schéma de la Figure 5.

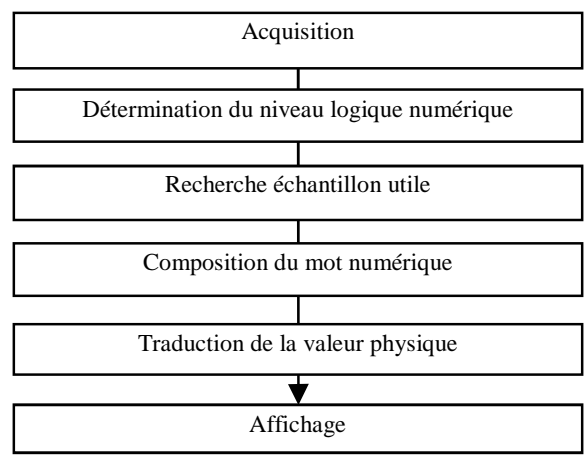

Fig. 5 : Organigramme logiciel.

Un exemple de VI de l'ensemble anémomètre et girouette est présenté Figure 6.

(a)

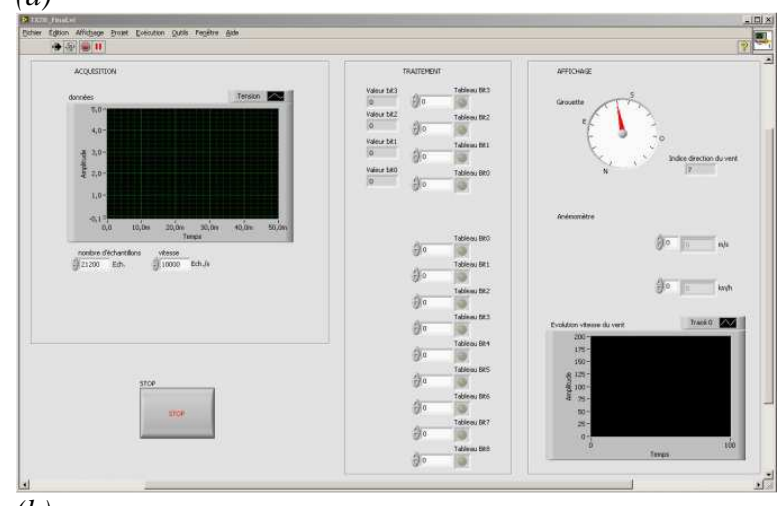

(b)

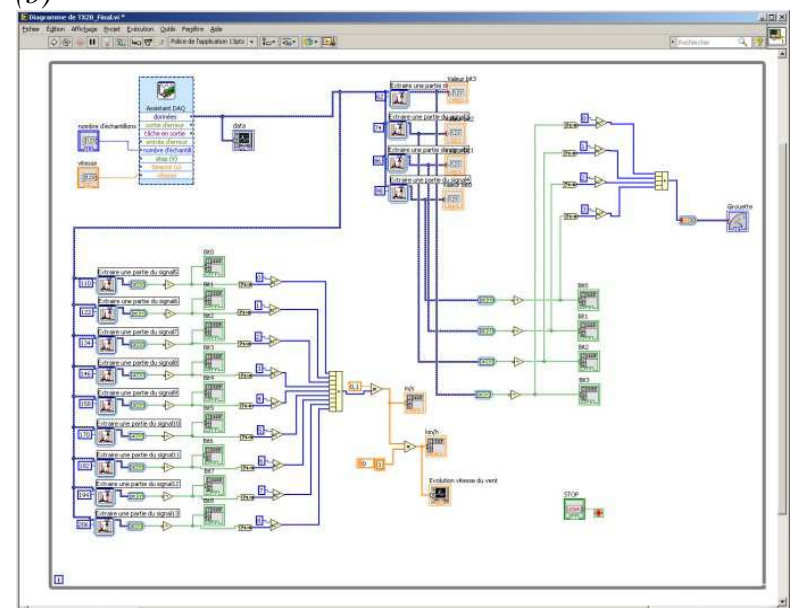

Fig. 6 : Exemples de (a) face avant et de (b) diagramme pour l'ensemble anémomètre et girouette.

\subsection{Influence des bruits d'alimentation}

En aparté des mesures précédemment décrites, les étudiants prennent conscience du phénomène de bruit apporté par l'alimentation du système. Trois sources d'alimentation sont à leur disposition : alimentation secteur à découpage, alimentation série à base de transformateur et de régulateur, batterie.
L'emploi successif de chacune de ces sources (bruitées dans des domaines fréquentiels différents) permet d'appréhender l'influence des bruits des alimentations dans la mesure. L'étudiant apprend alors à y remédier logiciellement via un filtrage des paquets d'échantillons.

\section{PEDAGOGIE ET RETOUR D'EXPERIENCES}

\subsection{Pédagogie}

Les objectifs pédagogiques de ce T.P. s'appuient sur la taxonomie de Bloom qui est un modèle pédagogique proposant une classification des niveaux d'acquisition des connaissances (Figure 7). Elle organise l'information de façon hiérarchique, de la simple restitution des faits jusqu'à la manipulation des concepts et peut être résumée en six niveaux, chaque niveau supérieur englobant les niveaux précédents [8] [9].

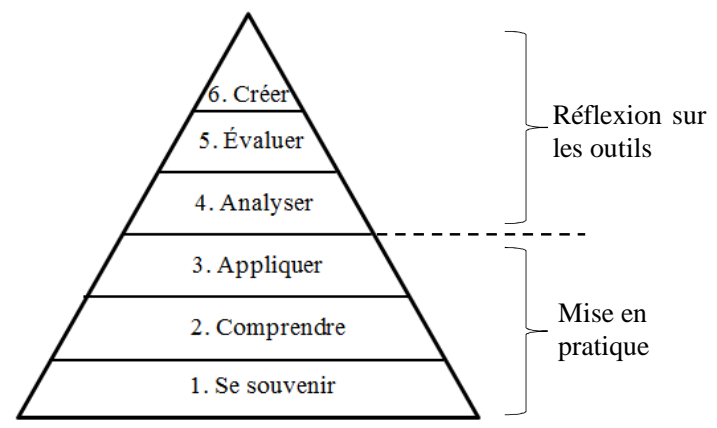

Fig. 7 : Taxonomie de Bloom.

Le Tableau 1 associe des exemples de compétences aux niveaux de Bloom correspondants.

Tableau 1 : Exemples de compétences en fonction des niveaux de Bloom associés.

\begin{tabular}{|l|c|}
\hline \multicolumn{1}{|c|}{ Compétences } & $\begin{array}{c}\text { Niveau de } \\
\text { Bloom }\end{array}$ \\
\hline $\begin{array}{l}\text { Restituer les connaissances en électro- } \\
\text { nique, acquisition et traitement des don- } \\
\text { nées. }\end{array}$ & 1 \\
\hline $\begin{array}{l}\text { Appréhender les pièges de la mise en } \\
\text { œuvre en mesure (désadaptation } \\
\text { d'impédance entre quadripôles). }\end{array}$ & 1 \\
\hline $\begin{array}{l}\text { Comprendre l'architecture de la carte } \\
\text { électronique (documentation technique } \\
\text { des composants). }\end{array}$ & 2 \\
\hline $\begin{array}{l}\text { Comprendre les montages à base d'AOP } \\
\text { (amplificateur non inverseur, suiveur, } \\
\text { amplificateur différentiel, soustracteur) et } \\
\text { les modes (différentiel, mode commun). }\end{array}$ & 2 \\
\hline $\begin{array}{l}\text { Appliquer les équations des réseaux de } \\
\text { résistances (pont diviseur de tension, } \\
\text { théorème de Millmann), le gain en ten- } \\
\text { sion des montages à base d'AOP, les } \\
\text { formules trouvées dans les documenta- } \\
\text { tions techniques. }\end{array}$ & \\
\hline
\end{tabular}




\begin{tabular}{|l|c|}
\hline $\begin{array}{l}\text { Visualiser et comprendre à l'aide d'un } \\
\text { oscilloscope les signaux des capteurs (en } \\
\text { particulier pour l'anémomètre/girouette). }\end{array}$ & 4 \\
\hline $\begin{array}{l}\text { Analyser la forme périodique ou non du } \\
\text { signal issu du luxmètre pour en déduire sa } \\
\text { provenance et ce, en fonction de la source } \\
\text { d'éclairement : néon, lumière extérieure, }\end{array}$ & 4 \\
$\begin{array}{l}\text { DEL. } \\
\text { Déterminer la meilleure source } \\
\text { d'alimentation pour l'électronique de } \\
\text { mesure (génération de bruit). }\end{array}$ & 5 \\
\hline $\begin{array}{l}\text { Créer un ensemble logiciel pour } \\
\text { l'instrumentation (procédé et interface }\end{array}$ & 6 \\
graphique). & 6 \\
\hline
\end{tabular}

\subsection{Retour d'expériences}

Le T.P. météo fonctionne depuis 2010 pour les mesures de température, de pression atmosphérique et de quantité de lumière et depuis 2013 pour les mesures de direction et de vitesse du vent (environ 90 étudiants). Nous avons demandé aux étudiants ayant suivi ce T.P. cette année de remplir un questionnaire anonyme à la fin des séances. L'objectif de ce questionnaire est double : avoir un retour des étudiants et donner une note de satisfaction globale. Six questions ont été posées et notées de « 1 » à « 4 », les notes « 1 » et « 4 » représentant respectivement les notes minimale et maximale obtenues pour chaque question. Les étudiants pouvaient également proposer des améliorations. Le tableau 2 synthétise les résultats.

Tableau 2 : Résultats de l'évaluation du T.P. météo.

\begin{tabular}{lcccc}
\hline \multicolumn{1}{c}{ Question } & Non=1 & $\begin{array}{c}\text { Plutôt } \\
\text { Non=2 }\end{array}$ & $\begin{array}{c}\text { Plutôt } \\
\text { Oui= 3 }\end{array}$ & Oui=4 \\
\hline $\begin{array}{l}\text { 1. La variété des connaissances acquises vous semble-t-elle suffisante (différents } \\
\text { capteurs, chaine de mesure, LabVIEW...)? }\end{array}$ & $0 \%$ & $0 \%$ & $82,3 \%$ & $17,7 \%$ \\
$\begin{array}{l}\text { 2. La résolution des verrous techniques vous semble-t-elle suffisamment approfon- } \\
\text { die (étude des schémas électroniques, recherches des informations fabricants...)? }\end{array}$ & $0 \%$ & $0 \%$ & $82,3 \%$ & $17,7 \%$ \\
$\begin{array}{l}\text { 3. Le travail, à vocation d'autonomie, est-il suffisamment guidé? } \\
\text { 4. L'enseignement a-t-il contribué à élargir vos techniques en informatique d'ins- } \\
\text { trumentation? }\end{array}$ & $0 \%$ & $29 \%, 4 \%$ & $23,5 \%$ & $47,1 \%$ \\
$\begin{array}{l}\text { 5. L'enseignement a-t-il contribué à élargir votre sens critique ou votre approche } \\
\text { pratique de la mesure? }\end{array}$ & $0 \%$ & $29,4 \%$ & $52,9 \%$ & $17,7 \%$ \\
6. L'enseignement a-t-il contribué à élargir vos échanges avec les autres étudiants ? & $0 \%$ & $17,6 \%$ & $35,3 \%$ & $47,1 \%$ \\
\hline \hline
\end{tabular}

\section{CONCLUSION}

Le T.P. présenté dans cet article propose à des étudiants de licence professionnelle SiMCo de travailler sur une chaine complète d'instrumentation allant du capteur à la présentation des données traitées. Au cours de ce T.P., l'étudiant rencontre des domaines variés de la mesure physique (électronique, physique, informatique, traitement du signal...) et doit donc faire converger l'ensemble des notions d'électronique, de physique, d'informatique industrielle... enseignées séparément par ailleurs vers un même but : la réalisation de ce T.P.

L'étudiant est confronté à un système ouvert et modulable et pour remplir les objectifs du T.P., il est amené à faire des choix. C'est par l'autonomie «guidée » qu'il va conforter son expérience dans ce domaine. Cette approche pédagogique qui donne une grande part à l'autonomie, prépare l'étudiant à mettre en œuvre des capacités techniques au spectre élargi dans des situations qu'il rencontrera très certainement dans sa vie professionnelle future. Le dialogue permanent et le sondage nous fait entrevoir une forte adhésion de leur part à cette méthode, c'est-à-dire autonomie relative, échange et variété technique.
Pour compléter ce T.P., il est envisagé d'ajouter un nouveau capteur comme une humidistance et de prévoir la mise en forme du signal anémométrique dégradé par la longueur du câble.

\section{Bibliographie}

[1] National Instruments, "NI USB-6008/6009 User Guide and Specifications",

[2] J. Hay, "L'art de l'amplificateur opérationnel : le composant et ses principales utilisations", Publitroni, ISBN 2 86661044 X (1995).

[3] G. Isabel, "Mesures et comptage : s'initier par la pratique", Editions Techniques et Scientifiques Françaises, ISBN 2100053280 (2002).

[4] National Semiconductor, "Datasheet LM335" (1995).

[5] OSRAM, "SFH 5711" (2013).

[6] Motorola, "Semiconductor Technical Data MPX 220 series", Rev. 9 (2002).

[7] john.geek.nz, http://www.john.geek.nz/2011/07/la-crossetx20-anemometer-communication-protocol/, site web (2011).

[8] B.S. Bloom, M.D. Englehart et al., "Taxonomy of educational objectives. Handbook 1: Cognitive domain", New York: McKay (1956).

[9] L.W. Anderson, D.R. Krathwohl et al., "A taxonomy for learning, teaching, and assessing: A revision of Bloom's Taxonomy of Educational Objectives", New York: Longman (2001). 